\title{
Thirty-Day Morbidity and Mortality after Simultaneous Bilateral Total Knee Arthroplasty
}

\author{
CC Tai, FRCS Orth, SH Tan, MBBS, NA Misnan, MBBS, HY Nam, MSc, SK Choon, FRCS (Edin) \\ Department of Orthopaedic Surgery, University Malaya Medical Centre, Kuala Lumpur, Malaysia
}

\begin{abstract}
The safety of simultaneous bilateral total knee arthroplasty (TKA) remains controversial. The objective of the current study was to investigate perioperative morbidity and mortality rates within 30 days of simultaneous bilateral TKA. A detailed analysis of medical, surgical and anaesthesia records of 183 consecutive patients who underwent total knee arthroplasty between 2002 and 2006 was performed. The mean age of the patients was 67.6 years old. More than $80 \%$ had one or more co-morbidities, but none of them had ASA score greater than class 2. The mean hospital stay was 10 days, and the mean surgical time 156 minutes. Less than half of the patients $(42.6 \%)$ required blood transfusion. The rate of perimorbidity was $15.3 \%$ and there was no mortality in this series. We believe that simultaneous bilateral total knee arthroplasty is a safe and cost effective option for our patients, provided that patients are selected and informed appropriately.
\end{abstract}

Key Words:

Bilateral total knee arthroplasty, Morbidity, Mortality

\section{INTRODUCTION}

Total knee arthroplasty (TKA) is a common orthopaedic procedure that is considered highly successful and safe, with very low rates of perioperative complications and death. However, there remains concern associated with simultaneous bilateral TKA. Several studies have reported increased risk of serious complications or even death when bilateral TKA are done simultaneously ${ }^{1,2}$, whereas other investigators have found that the rate of complications associated with simultaneous bilateral TKA is the same as or lower than that associated with unilateral TKA ${ }^{3,4}$.

In Malaysia, due to the increased accessibility to affordable and success TKA, more and more patients are requesting this procedure. Many of these patients have bilateral knee arthritis requiring operative intervention on both knees. The surgeon and patient are then faced with the decision of whether to proceed with a staged bilateral TKA, performed as two unilateral TKA procedures on separate days, or a simultaneous bilateral TKA. It is therefore important to ascertain the risks and complications associated with simultaneous TKA in this country. In addition, the current published literature is based on the western population in well-established arthroplasty centres. At present, there is no published data on post-operative morbidity and mortality after simultaneous bilateral TKA in an Asian arthroplasty centre in general, and in Malaysia in particular.

This study was therefore undertaken to assess the early complications and mortality occurring within 30 days of simultaneous bilateral TKA in our medical centre and to compare the outcome with that of other published reports, especially those from Western institutions. We also sought to identify any risk factors associated with increased morbidity and mortality of simultaneous bilateral TKA.

\section{MATERIALS AND METHODS}

The operative database in University Malaya Medical Centre (UMMC) was searched to retrospectively identify all patients who had undergone simultaneous bilateral TKA between June 2002 and June 2006. Patients were excluded if the procedure was a revision surgery, a two-stage procedure for septic arthritis, or an additional procedure was performed under the same anaesthesia.

The medical records of the patients who fulfilled the selection criteria were reviewed for demographic, hospital and surgical information including pre-operative diagnosis, duration of operation and hospital stay, method of anaesthesia, the American Society of Anaesthesiology (ASA) classification ${ }^{5}$ and post-operative blood transfusion. Risk factors such as body mass index and medical comorbidities were also noted.

The bilateral TKA were performed under a single (general or regional) or combined anaesthetic. The two procedures were either done simultaneously by two surgical teams or by a single surgical team in a sequential fashion - the procedure was started on the second knee after the first prosthesis was implanted but before the first knee was closed. All

Corresponding Author: Tai Cheh Chin, Department of Orthopaedic Surgery, University of Malaya, 50603 Kuala Lumpur, Malaysia Email: tai.chehchin@gmail.com 
procedures were performed under tourniquet control. An intramedullary alignment rod was utilized for femoral alignment, and an external tibial guide was used for tibial alignment. Either a cruciate-retaining, posterior stabilized, or rotating-platform prosthesis (DePuy Johnson and Johnson, Warsaw, Indiana) was used. All of the femoral and tibial components were fixed with polymethylmethacrylate.

All patients were cared for using a standardized postoperative TKA clinical protocol, which did not include any chemical DVT prophylaxis unless patients were known to have previous history of DVT. Patients were mobilized on the first postoperative day and were generally discharged when they were considered to be safe in the home environment and were able to achieve satisfactory range of motion $\left(0^{\circ}\right.$ to $\left.90^{\circ}\right)$. After discharge, the patients were evaluated in the outpatient clinic at 2 weeks, 4 weeks, 3 months, one year, and annually thereafter. For purposes of this study, post-operative events up to 30 days were evaluated to identify any complications or mortality brought about by the TKA procedure. A thirty-day perioperative time limit was selected on the assumption that a shorter time-period might have led to the exclusion of deaths that were directly related to the surgery whereas a longer time-period might have led to the inclusion of deaths that were not attributable to the operation.

A deep infection was considered any infection that occurred inside the knee joint and required removal of the prosthesis. A superficial infection was any infection of the skin that responded well to antibiotics with no residual sequelae ${ }^{4}$.

\section{Statistical Analysis}

Single risk factors were analyzed with either the chi-square test or the Fisher exact test. Multivariate analysis was performed with use of a logistic regression model. Risk factors were retained in the model if they were significant $(p$ $<0.05$ ). During regression modelling, two-way interactions were studied for all combinations of risk factors. After completion of the modelling, the relative risk for each combination of risk factors was estimated with 95\% confidence intervals.

\section{RESULTS}

\section{Demographic Data}

A total of 183 patients who had bilateral TKA between June 2002 and June 2006 met the criteria and were included in this study. One hundred and sixty two of these patients were women, and 21 were men. The average age of the patients was 67.6 (range, 35-87). The predominant diagnosis was bilateral osteoarthritis (in $95.1 \%$ of subjects). The mean weight of patients was $66.8 \mathrm{~kg}$ (range, 41-105). According to body mass index (BMI), $40.9 \%$ of the patients were found to be obese $(\mathrm{BMI}>30)$ and $3.3 \%$ were morbidly obese $(\mathrm{BMI}>$ 40) (Table I).

\section{Pre-operative morbidity and Mobility}

Among the patients in this study, only 36 patients presented with no co-morbidities. All other patients had at least one or more preoperative co-morbidities (Table II). The most common co-morbidity was hypertension, which affected 108 patients $(59.0 \%)$. All but one of those patients were receiving antihypertensive treatment. The next most common comorbidity was diabetes mellitus $(14.8 \%)$ followed by hyperlipidaemia $(8.7 \%)$. There were only 9 smokers in this group of patients (Table III). Using the ASA system, most patients were categorized as being in Class $2(62.8 \%)$ and 41 patients $(22.4 \%)$ were classified as being in Class 1 . There was no clear documentation of the ASA status in the remaining 27 patients.

The pre-operative ambulatory status of all but 46 patients was documented. Seventy-one $(38.8 \%)$ patients were able to walk without aid. Fifty-five patients were able to move around with canes $(22.9 \%)$ or walking frames $(7.1 \%)$, and 11 $(6.0 \%)$ were confined to wheelchairs.

Post-operatively upon discharge, all patients were capable of ambulation with walking frames. At the latest follow up, 180 patients $(98.4 \%)$ were able to walk without any aids, one patient $(0.5 \%)$ with a cane and $2(1.1 \%)$ with walking frames. The three patients who required some assistance with walking suffered from other joints problems, including one with chronic back pain.

\section{Hospitalization and Surgical Details}

The mean hospital stay was $10.0 \mathrm{~d}$ (range, 4-44 d). Remember that patients were discharged when they were able to ambulate independently with walking frames and capable of active extension and flexion up to $90^{\circ}$.

Total surgical time averaged 156.9 minutes (range, 60-240 minutes) in this study. There were 38 sequential procedures and the remaining 145 knee replacements were carried out simultaneously. The average duration for sequential operations was 198.5 minutes (range, 93-240 minutes) whereas the latter group averaged at 142.9 minutes (range, 60-180 minutes). Seventy-eight patients (42.6\%) required blood transfusion after surgery and received an average of 2.5 units (range, 1-7 units).

There were some variations in the method of anaesthesia. General anaesthesia (GA) was most commonly used (60.1\%) while spinal anaesthesia was given to $22.9 \%$ of the patients. Twenty-three patients (12.6\%) received epidural anaesthesia. Occasionally, combined GA and epidural (1.6\%) or combined spinal and epidural $(2.7 \%)$ were given.

\section{Mortality and Morbidity}

In this study group, there were no deaths within the 30-day time period after surgery. One hundred and fifty five patients $(84.7 \%)$ did not experience any post-operative 
Table I: Characteristics of patients who underwent simultaneous bilateral total knee arthroplasty. ( $n=$ number of patients)

\begin{tabular}{|c|c|}
\hline Characteristics & \\
\hline Mean age (range) & 67.6y (range, $35-87 y)$ \\
\hline Gender & \\
\hline Women $(n, \%)$ & $162(88.5)$ \\
\hline Male $(n, \%)$ & $21(11.5)$ \\
\hline Ethnicity & \\
\hline Malays (n, \%) & $26(14.2)$ \\
\hline Chinese $(n, \%)$ & $128(69.9)$ \\
\hline Indians $(n, \%)$ & $28(15.3)$ \\
\hline Other $(n, \%)$ & $1(0.5)$ \\
\hline Body mass index classification & \\
\hline Obese $(n, \%)$ & 75 (40.9) \\
\hline Morbidly Obese (n, \%) & $6(3.3)$ \\
\hline Preoperative Diagnosis & \\
\hline Osteoarthritis (n, \%) & $174(95.1)$ \\
\hline Rheumatoid Arthritis ( $\mathrm{n}, \%$ ) & $9(4.9)$ \\
\hline Mobility Status Pre-op & \\
\hline Without aid $(n, \%)$ & $71(38.8)$ \\
\hline Cane $(n, \%)$ & $42(22.9)$ \\
\hline Walking frame $(n, \%)$ & $13(7.1)$ \\
\hline Wheel chair $(n, \%)$ & $11(6.0)$ \\
\hline Unknown $(n, \%)$ & $46(25.1)$ \\
\hline Method of anaesthesia & \\
\hline General anaesthetic, GA (n, \%) & $110(60.1)$ \\
\hline Spinal $(n, \%)$ & $42(22.9)$ \\
\hline Epidural $(n, \%)$ & $23(12.6)$ \\
\hline GA and epidural ( $n, \%)$ & $3(1.6)$ \\
\hline Spinal and epidural ( $n, \%)$ & $5(2.7)$ \\
\hline $\begin{array}{l}\text { American Society of Anaesthesiology } \\
\text { (ASA classification) }\end{array}$ & \\
\hline ASA $1(n, \%)$ & $41(22.4)$ \\
\hline ASA $2(n, \%)$ & $115(62.8)$ \\
\hline Unknown (n, \%) & $27(14.7)$ \\
\hline
\end{tabular}

Table Il: Number of existing co-morbidities among patients who underwent simultaneous bilateral total knee arthroplasty.

\begin{tabular}{|l|c|}
\hline Number of Co-morbidities & Patients $(\mathbf{n}, \%)$ \\
\hline No Co-morbidities & $36(19.7)$ \\
One co-morbidity & $46(25.1)$ \\
Two co-morbidities & $49(26.8)$ \\
Three or more co-morbidities & $52(28.4)$ \\
Total & $183(100)$ \\
\hline
\end{tabular}

Table III: Co-morbidities observed in patients with simultaneous bilateral total knee arthroplasty

\begin{tabular}{|c|c|}
\hline Co-morbidities & Number of Patients \\
\hline \multicolumn{2}{|l|}{ Cardiac } \\
\hline Hypertension & 108 \\
\hline Ischaemic Heart Disease & 10 \\
\hline Ventricular hypertrophy & 2 \\
\hline Cardiomyopathy & 1 \\
\hline Aortic aneurysm & 1 \\
\hline \multicolumn{2}{|l|}{ Pulmonary } \\
\hline Asthma & 7 \\
\hline Bronchitis & 2 \\
\hline Pulmonary emboli & 1 \\
\hline Pneumonia & 1 \\
\hline \multicolumn{2}{|l|}{ Haematological } \\
\hline Lymphoma & 1 \\
\hline \multicolumn{2}{|l|}{ Endocrine } \\
\hline Diabetes & 27 \\
\hline Hypothyroidism & 6 \\
\hline \multicolumn{2}{|l|}{ Neurological } \\
\hline Stroke & 3 \\
\hline Parkinson's & 3 \\
\hline Alzheimer's & 1 \\
\hline \multicolumn{2}{|l|}{ Musculoskeletal } \\
\hline Spinal problems & 6 \\
\hline Spondylosis & 5 \\
\hline History of septic knee arthritis & 2 \\
\hline Myasthenia gravis & 1 \\
\hline Osteoporosis & 2 \\
\hline Rheumatoid arthritis & 12 \\
\hline Gout / pseudogout & 4 \\
\hline \multicolumn{2}{|l|}{ Others } \\
\hline Hypercholesterolaemia & 3 \\
\hline Hyperlipidaemia & 16 \\
\hline Thalassaemia & 1 \\
\hline Smoking & 9 \\
\hline
\end{tabular}

Table IV: Complications observed in patients within the 30-day time period after simultaneous bilateral total knee arthroplasty. (Some patients developed more than one complication.)

\begin{tabular}{|l|c|}
\hline Complication & Number of Patients \\
\hline Death within 30 days of surgery & 0 \\
Atrial fibrillation & 1 \\
Myocardial infarction & 0 \\
Respiratory failure & 2 \\
Pneumonia & 2 \\
Pulmonary embolus & 0 \\
Deep vein thrombosis & 4 \\
Fat emboli syndrome & 0 \\
Cerebrovascular accident & 1 \\
Peroneal nerve palsy & 3 \\
Urinary retention & 2 \\
Acute renal failure & 2 \\
Urinary tract infection & 3 \\
Electrolyte imbalance & 5 \\
Paralytic lleus & 0 \\
Wound dehiscence & 3 \\
Septicaemia & 1 \\
Superficial infection & 10 \\
Deep infection & 2 \\
Intraoperative fracture & 1 \\
Pressure sore & 2 \\
\hline
\end{tabular}


complications. A list of the complications encountered is presented in Table IV. There were 16 readmissions documented, the majority (12 patients) was due to infection. There were 10 cases of superficial infection and 2 cases of deep infection. The overall infection rate in bilateral TKA (366 total knee replacement) was therefore 3.3\%, and the deep infection rate $0.5 \%$. All infections were limited to a single knee joint only. No infection to the contralateral knee was reported in subsequent follow-up up to a minimum of 12 months.

Three patients (1.6\%) experienced initial peroneal nerve palsy post-operatively in a single knee joint. None complained of bilateral peroneal nerve palsy. All patients with peroneal nerve palsy resolved within 6 months after surgery, without further surgical intervention.

There was one incident of cardiac complications, (atrial fibrillation), and four known cases of deep vein thrombosis (DVT). These patients were treated with medication and did not suffer any sequelae. There were no reported cases of myocardial infarction, pulmonary embolism or fat embolism syndrome.

There were three cases of intensive care admission - two patients developed respiratory failure requiring ventilation, one due to aspiration pneumonia and the other one due to septicaemia. A third intensive care unit admission was due to one patient diagnosed with brainstem stroke that developed within 24 hours after surgery.

Minor gastrointestinal complications, principally postoperative nausea and vomiting (13.7\%), and dizziness $(18.6 \%)$ were relatively common. In $5.5 \%$ of the patients, blood tests showed electrolyte imbalance that was easily corrected within a few days except for one patient who had persistent mild hyperkalaemia that persisted even after discharge.

There was no statistically significant difference between sequential and non-sequential bilateral total knee replacement in term of mortality, post-operative morbidity, requirement of blood transfusion after surgery, duration of hospitalization and patient mobility upon discharge from hospital. There was also no clear correlation between age, sex, ethnicity, BMI, number of existing co-morbidities, diagnosis and post-operative complications.

\section{DISCUSSION}

The suitability of TKA as a simultaneous bilateral procedure has been debated for many years and remains controversial. While TKA is considered extremely safe, a simultaneous bilateral procedure is undeniably more physically demanding for both the patient and the surgeon. Patients undergoing a TKA generally belong to the older age group and most have pre-operative co-morbidities, (as shown in this study, in which more than $80 \%$ of study subjects have at least one or more existing co-morbidities). This results in a potential increase in complications and mortality rate associated with surgery.

Parvizi et al investigated the mortality rate within thirty days after TKA and reported that the mortality rate after simultaneous bilateral TKA $(0.49 \%)$ was significantly higher than after unilateral TKA $(0.17 \%)$. However, it is important to note that less than $12 \%$ of their patients underwent bilateral TKA. Other investigators have shown that the incidence of mortality after simultaneous bilateral TKA was similar to unilateral TKA ${ }^{3,6}$. In our series of 183 patients, we have no mortality observed within 30 days after operation. One likely explanation is careful patient selection. All our patients who agree to have simultaneous bilateral TKA must undergo thorough medical screening to assess his/her medical fitness to undergo the procedure. Our anaesthetists routinely perform pre-operative assessment two weeks prior to surgery to exclude inappropriate candidates; hence most if not all of our patients were in either ASA class 1 or 2 . The other advantage of having the pre-operative assessment 2 weeks prior to surgery is that we are able to assess and optimise patient condition for the surgery at that time.

Rates of perioperative morbidity are of concern associated with simultaneous bilateral TKA. Several authors have shown that bilateral procedures increased the prevalence of fat emboli with resulting pulmonary and neurological sequelae $^{7}$. Other studies have reported a higher incidence of cardiovascular complications ${ }^{8}$, pulmonary emboli ${ }^{9}$, greater blood loss and postoperative blood transfusion requirements ${ }^{10}$. In our study, there were only two cases of cardiovascular complications. There were no reported cases of myocardial infarction despite the majority of the patients have one or more co-morbidities such as smoking, diabetes and hypertension, which predispose to increased cardiovascular risk. There were also no documented cases of pulmonary embolism. The prevalence of DVT following simultaneous bilateral TKA in our study was low, with only four cases of deep vein thrombosis (DVT) noted. This was consistent with the findings of a lower rate of DVT in simultaneous bilateral TKA in several studies ${ }^{10,11}$. In the current study, an identical postoperative DVT prophylaxis protocol consisting of compression stockings and early mobilization was implemented for all patients. Less than half of the patients required blood transfusion and the average number of units of blood transfused was 2.5 units for those who needed transfusion, a result comparable to unilateral TKA $^{12}$.

The total infection rate of $3.3 \%$ and deep infection rate of $0.5 \%$ also compares very favourably to other studies including unilateral TKA ${ }^{4,13}$. We therefore believe that the incidence of complications of simultaneous bilateral TKA is 
not greater than unilateral TKA, provided that there is appropriate patient selection and assessment. It is also interesting to note that there is no significant difference between the patients who have sequential and non-sequential bilateral total knee replacement in terms of perioperative morbidity.

One of the advantages of performing simultaneous bilateral TKA is the shorter total hospital stay compared with bilateral TKA performed on separate occasions. The average hospital stay of the patients with bilateral TKA in our institution was 10 days which is comparable to duration of hospital stay for patients who undergo unilateral TKA in many arthroplasty centres. The other added benefit is that patients are required to undergo only one anaesthesia and shorter total duration of anaesthesia compared to staged unilateral TKA. In addition, patients also spend less total time in rehabilitation therapy. All of these advantages may result in more cost-effective treatment ${ }^{3,11}$. In our centre all patients with symptoms severe enough to warrant TKA are offered a simultaneous procedure, $40 \%$ of our patients choose simultaneous TKA. With increased health care costs, and with more patients in this country presenting with bilateral knee problems, simultaneous bilateral TKA may provide a substantial cost saving for both the patients and hospitals.

This study did not demonstrate any clear evidence of risk factors associated with a significant increase in thirty-day morbidity. Factors investigated include age, sex, ethnicity, obesity, diagnosis and the number of existing co-morbidities. Patients aged 80 years or older have been reported to have higher incidences of complications ${ }^{2}$ while another study suggested an age of 70 years as the appropriate threshold to minimize risk ${ }^{14}$. However, our study did not show a correlation between age and the incidence of post-operative complications. Similarly, obesity including morbidly obesity was not a significant risk factor. However, it must be noted that only six patients fell into this category and a larger sample size is needed for a proper unbiased evaluation of this factor. The lack of evidence of the positive correlation between the number of co-morbidities in patients who underwent bilateral TKA and their post-operative complications suggest that perhaps the severity of each comorbidity, and not the number of existing co-morbidities, is more important as a factor leading to post-operative complications.

Limitations of this study include the inherent problems with a retrospective review such as information bias and lack of a control group. However, the current cohort consisted of consecutive, non-selected patients, and the sample size was relatively large. This is an ongoing study, and we hope to follow up with the patients over a longer period of time and present longer term morbidity (more than 30 days) and clinical outcome data including implant survival rates in the future.

\section{CONCLUSION}

The current study established that simultaneous bilateral TKA can be a safe and cost-effective option when patients are sufficiently symptomatic to warrant surgery in both knees. However, it is important to perform thorough preoperative assessment on these patients, and careful patient selection is crucial to ensure the success of the operation and avoidance of complications. We believe that simultaneous bilateral TKA does not subject patients to an unacceptable level of risk and the decision to proceed with simultaneous surgery should depend on patient preference through informed choice. 


\section{REFERENCES}

1. Parvizi J, Sullivan TA, Trousdale RT, Lewallen DG. Thirty-day mortality after total knee arthroplasty. J Bone Joint Surg Am. 2001; 83: 1157-61.

2. Lombardi AV, Mallory TH, Fada RA, Hartman JF, Capps SG, Kefauver CA, et al. Simultaneous bilateral total knee arthroplasties: who decides? Clin Orthop. 2001; 392: 319-29.

3. Bullock DP, Sporer SM, Shirreffs TG Jr. Comparison of simultaneous bilateral with unilateral total knee arthroplasty in terms of perioperative complications. J Bone Joint Surg Am. 2003; 85: 1981-6.

4. Ritter MA, Harty LD, Davis KE, Meding JB, Berend M. Simultaneous bilateral, staged bilateral, and unilateral total knee arthroplasty. A survival analysis. J Bone Joint Surg Am. 2003; 85: 1532-7.

5. American Society of Anesthesiologists. New classification of physical status. Anesthesiology 1963; $24: 111$.

6. Morrey BF, Adams RA, Ilstrup DM, Bryan RS. Complications and mortality associated with bilateral or unilateral total knee arthroplasty. J Bone Joint Surg Am. 1987 69: 484-8.

7. Dorr LD, Merkel C, Mellman MF, Klein I. Fat emboli in bilateral total knee arthroplasty. Predictive factors for neurologic manifestations. Clin Orthop. 1989;248:112-9.

8. Lynch NM, Trousdale RT, Ilstrup DM. Complications after concomitant bilateral total knee arthroplasty in elderly patients. Mayo Clin Proc. 1997; 72: 799-805.

9. Barrett J, Baron JA, Losina E, Wright J, Mahomed NN, Katz JN. Bilateral total knee replacement: staging and pulmonary embolism. J Bone Joint Surg Am. 2006; 88: 2146-51.

10. Cohen RG, Forrest CJ, Benjamin JB. Safety and efficacy of bilateral total knee arthroplasty. J Arthroplasty. 1997; 12: 497-502.

11. Jankiewicz JJ, Sculco TP, Ranawat CS, Behr C, Tarrentino S. One-stage versus 2-stage bilateral total knee arthroplasty. Clin Orthop. 1994; 309: 94-101.

12. Bierbaum BE, Callaghan JJ, Galante JO, Rubash HE, Tooms RE, Welch RB. An analysis of blood management in patients having a total hip or knee arthroplasty. J Bone Joint Surg Am. 1999; 81-A: 2-6.

13. Hutchinson JRM, Parish EN, Cross MJ. A comparison of bilateral uncemented total knee arthroplasty: simultaneous or staged? $J$ Bone Joint Surg Br. 2006; 88-B: 40-3.

14. Oakes D, Hanssen A. Bilateral total knee replacement using the same anesthetic is not justified by assessment of the risks. Clin Orthop. 2004; 428: 87-91. 